\title{
Efficient BRDF Sampling Using Projected Deviation Vector Parameterization
}

Tanaboon Tongbuasirilai, J onas Unger and Murat Kurt

\section{Conference article}

Cite this conference article as:

Tongbuasirilai, T., Unger, J ., Kurt, M. Efficient BRDF Sampling Using Projected Deviation Vector Parameterization, In 2017 IEEE International Conference on Computer Vision Workshops (ICCVW), Institute of Electrical and Electronics Engineers (IEEE); 2017, pp. 153-158. ISBN: 978-1-5386-1034-3

DOI: https:// doi.org/ 10.1109/ ICCVW.2017.26

IEEE International Conference on Computer Vision Workshops, ISSN: 2473-9936, 2016

Copyright: IEEE

The self-archived postprint version of this conference article is available at Linköping University Institutional Repository (DiVA):

http:// urn.kb.se/ resolve?urn=urn:nbn:se:liu:diva-145821 


\section{Efficient BRDF Sampling Using Projected Deviation Vector Parameterization}

\author{
Tanaboon Tongbuasirilai \\ Linköping University \\ tanaboon.tongbuasirilaidiu.se
}

\author{
Jonas Unger \\ Linköping University \\ jonas.unger@liu.se
}

\author{
Murat Kurt \\ International Computer Institute \\ Ege University \\ murat.kurteege.edu.tr
}

\begin{abstract}
This paper presents a novel approach for efficient sampling of isotropic Bidirectional Reflectance Distribution Functions (BRDFs). Our approach builds upon a new parameterization, the Projected Deviation Vector parameterization, in which isotropic BRDFs can be described by two $1 D$ functions. We show that BRDFs can be efficiently and accurately measured in this space using simple mechanical measurement setups. To demonstrate the utility of our approach, we perform a thorough numerical evaluation and show that the BRDFs reconstructed from measurements along the two $I D$ bases produce rendering results that are visually comparable to the reference BRDF measurements which are densely sampled over the $4 D$ domain described by the standard hemispherical parameterization.
\end{abstract}

\section{Introduction}

The scattering of light at a surface, described by the Bidirectional Reflectance Distribution Function (BRDF) [8], is the fundamental aspect in most computer vision and graphics applications. Accurate descriptions of material properties such as color, reflectance and texture are key components in photo realistic image synthesis. In computer vision it is often necessary to model, represent and process the material characteristics and scattering behavior in order to perform higher level semantic analysis of scenes captured using image based methods or accurate reconstruction of 3D shapes. These applications have driven the research and development of a large set of methods and techniques for measuring and modeling BRDFs and Spatially Varying BRDFs (SVBRDFs) such that they can be efficiently used for analysis and synthesis of material properties, for an overview see [3]. A difficult challenge, however, is that most BRDF measurement techniques are very time consuming as the radiance scattered at the surface needs to be densely sampled over the 4D space of incident $\omega_{i}$ and scattered (outgoing) $\omega_{o}$ directions.

In this paper, we describe a novel BRDF parameteriza- tion called Projected Deviation Vector (PDV) parameterization, which allows isotropic BRDFs to be accurately represented as a multiplication of two 1D basis functions. We show how this property can be exploited to enable efficient and accurate measurement of isotropic BRDFs in a single planar slice of the standard 4D hemispherical parameterization $\left(\omega_{i}, \omega_{o}\right)$. We evaluate our method using the MERL BRDF data base presented in [7] as a reference, and discuss how simple but accurate measurement devices can be constructed.

\section{Background}

Accurate measurement and modeling of BRDFs and SVBRDFs is an extensively researched field, for an overview see [3]. Due to the flexibility most approaches for BRDF measurements still build on point sampling and gonio-reflectometers such as $[1,2]$. The advantage of gonio-reflectometers is that the mechanics and optics are relatively simple as only a light source, a digital light sensor such as a camera and some motors are required; all of which can be bought off-the-shelf. The downside, however, is that it may take hours or even days to densely sample the full 4D BRDF.

In the pioneering work described in [13], Ward developed a setup consisting of a hemispherical mirror and a camera with a fisheye lens to simultaneously capture all outgoing directions as a light source was moved over all incident directions to efficiently capture the full BRDF. The widely used MERL BRDF data base described in [7], used as reference in this paper, was similarly to the work by Marschner et al. [6] captured using the same principles, but instead of using a hemispherical mirror to sample all reflected rays the physical material samples were shaped as spheres. To capture the BRDF each spherical material was imaged using a digital camera capturing all outgoing directions as a light source was moved around the sample. The MERL BRDF data consists of 100 isotropic materials and is stored in the form of the so called Half-Diff parameterization developed by Rusinkiewicz [12]. Each material was sampled densely and kept with resolution of $90 \times 90 \times 180$ 


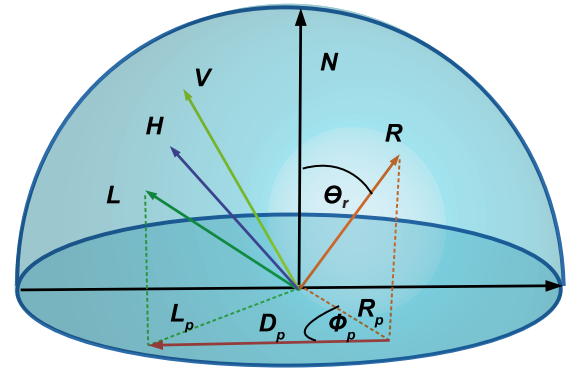

Figure 1. The projected deviation vector parameterization is formed by the projected deviation vector, $D_{P}$. The $D_{P}$ vector is the vector between the projected reflection vector, $R_{P}$ and the projected light vector, $L_{P}$, on the unit disk. The PDV parameterization consists of three parameters, $\left(\theta_{r}, d_{p}, \phi_{p}\right)$. $\theta_{r}$ is the zenith angle of the reflection vector, $R . d_{p}$ is the length of $D_{P}$ vector. $\phi_{p}$ is the azimuthal angle between $R_{P}$ and $D_{P}$.

for $\left(\theta_{h}, \theta_{d}, \phi_{d}\right)$ angles.

More recently attention has been put towards developing more efficient parameterizations, factorization methods, and in-depth analysis of efficient basis representations. The work by Romeiro et al. presented in [11] proposes a method where the Half-Diff parameterization, [12], is used to capture and represent isotropic BRDFs as a 2D reflectance function. By analyzing BRDF data bases, Xu et al. [14] and Nielsen et al. [9] developed approaches based on Principle Component Analysis (PCA), [4], of the MERL BRDF data base.

The PDV parameterization described in this paper is inspired by the work described by Löw et al. [5] and their study of the ABC BDRF models. As our main contribution, we show how the separability of isotropic BRDFs into two 1D basis functions can be exploited to develop fast measurement methods. For simplicity, we rely on traditional point sampling but we believe that the PDV parameterization could be used as the underlying representation to improve optimal sampling methods and BRDF reconstruction from basis representations such as PCA as described by Nielsen et al. [9]. Similarly to the 2D representation presented by Romeiro et al. [11], we believe that the PDV parameterization also could be used for BRDF inference from data captured in the wild.

\section{PDV Parameterization}

The PDV parameterization of BRDFs consists of three parameters $\left(\theta_{r}, d_{p}, \phi_{p}\right)$. These three parameters are related to the incident and outgoing vectors $\left(\omega_{i}, \omega_{o}\right)$ as shown in Figure 1. $\theta_{r}$ is the zenith angle of the perfect reflection of outgoing vector, $\omega_{o} . d_{p}$ is the length of the deviation vector, $D_{p}$. The deviation vector is formed by the projected vectors of both incoming and reflection vectors. The third parameter, $\phi_{p}$, is the azimuthal angle between the deviation vector and the $R_{p}$ vector. Algorithm 1 and 2 provide the pseudocode for converting between $\left(\omega_{i}, \omega_{o}\right)$ parameters and the PDV parameters.

$$
\begin{aligned}
& \text { Input: }\left(\theta_{i}, \phi_{i}, \theta_{o}, \phi_{o}\right) \\
& \text { Result: return }\left(\theta_{r}, d_{p}, \phi_{p}\right) \\
& \theta_{r}=\theta_{o} \\
& \phi_{i}=\phi_{i}-\phi_{o} \\
& \phi_{o}=0.0 \\
& R_{p}=\left(\sin \left(\theta_{o}\right) \cos \left(\phi_{o}+\pi\right), \sin \left(\theta_{o}\right) \sin \left(\phi_{o}+\pi\right)\right) \\
& L_{p}=\left(\sin \left(\theta_{i}\right) \cos \left(\phi_{i}\right), \sin \left(\theta_{i}\right) \sin \left(\phi_{i}\right)\right) \\
& D_{p}=L_{p}-R_{p} \\
& d_{p}=\operatorname{len}\left(D_{p}\right) \\
& \phi_{p}=\operatorname{atan} 2\left(D_{p} . y, D_{p} . x\right)
\end{aligned}
$$

Algorithm 1: Converting $\left(\omega_{i}, \omega_{o}\right)$ to PDV parameters.

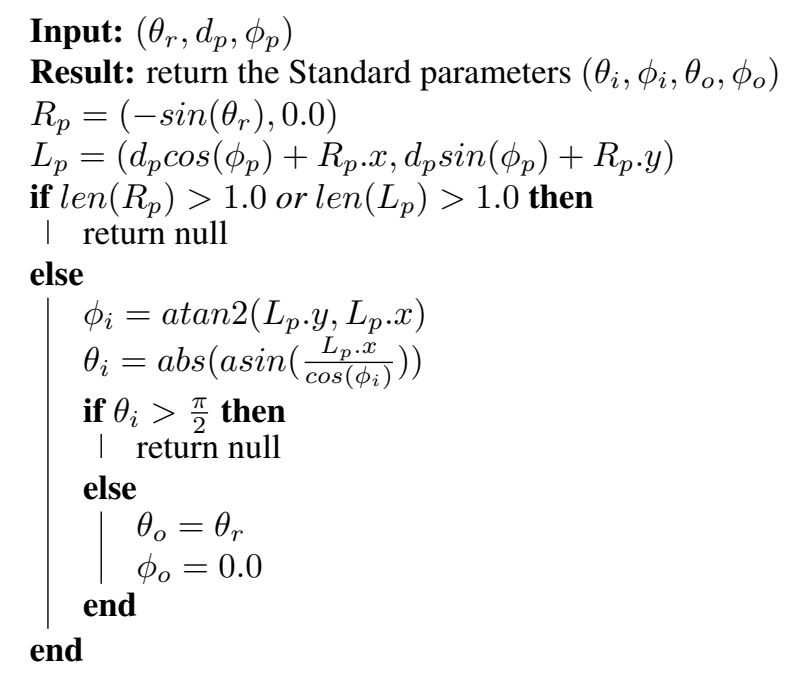

Algorithm 2: Converting PDV parameters to $\left(\omega_{i}, \omega_{o}\right)$.

\subsection{Parameter Sampling}

The $\theta_{r}$ and $\phi_{p}$ parameters are in the angular domain and can be efficiently sampled with evenly distributed samples over the parameter domain. However, as the $d_{p}$ parameter describes the shape of the BRDF lobe, linear sampling leads to an inefficient parameterization. Figure 2 shows the BRDF values of specific $\theta_{r}$ and $\phi_{p}$ along $d_{p}$ dimension. Most of the BRDF values outside of the specular region are relatively low and for many materials almost flat, and can be represented with only a small number of samples as compared to the specular peak where a higher sample density is required.

To compute an efficient sampling distribution along the $d_{p}$ parameter, we use the inversion method. Using all measurements in the MERL data base, [7], we linearly sampled the BRDF data in PDV space with 2000 evenly distributed samples along $d_{p}$ over its parameter range $[0,2)$. All of the sampled BRDFs are then summed using Equation 1: 


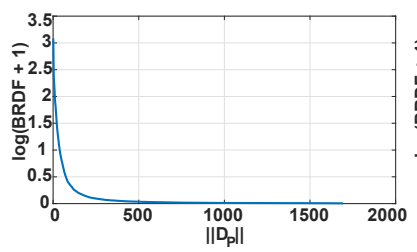

(a) alum-bronze BRDF

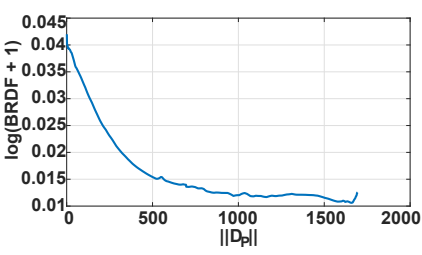

(b) blue-rubber BRDF
Figure 2. The figures contain examples of BRDF plots in the PDV parameterization. Both BRDFs are from a fixed angle of $\theta_{r}=45^{\circ}$ and $\phi_{p}=0^{\circ}$. Vertical axis is BRDF values scaled by logarithmic function and horizontal axis is the index of $d_{p}$ values. The left figure shows the BRDF plot of alum-bronze which represents the class of glossy materials. The right figure shows the BRDF plot of blue-rubber which represents the class of diffuse materials. It is apparent that BRDFs in the PDV parameterization aligned mostly around small $d_{p}$, i.e. around the specular peak.

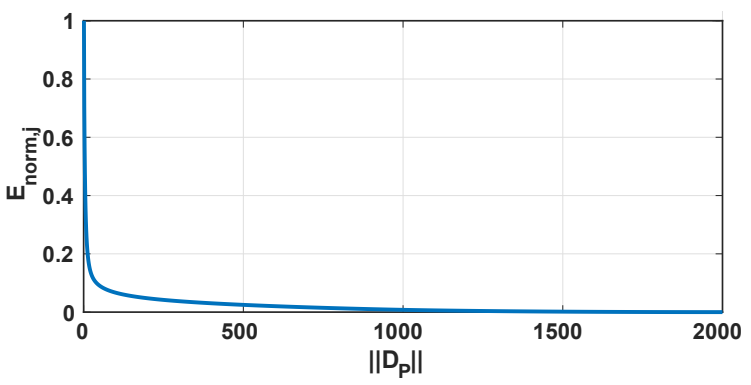

Figure 3. The sampling distribution along the $d_{p}$ parameter is computed to be inversely proportional to the $E_{n o r m, j}$ distribution computed as the mean over all materials in the MERL BRDF data base.

$$
E_{j}=\frac{\sum_{m}^{M} \sum_{i} \sum_{k} \rho_{m}(i, j, k)}{M},
$$

where $E_{j}$ is an element in a vector $E=\left\{E_{j} \mid j=\right.$ $1,2, \ldots, 2000\}, \rho_{m}$ is the BRDF value of $m$ th material and $M$ is the number of BRDFs in the data base.

We then normalize $E_{j}$ for the inversion method by $E_{n o r m, j}=\frac{E_{j}}{\sum E_{j}}$. The non-linear sample distribution along $d_{p}$ is then computed to be inversely proportional to the normalized mean distribution, $E_{\text {norm }, j}$, computed from BRDFs in the MERL data base as illustrated in Figure 3. For the experiments in this paper we used 90 non-linearly distributed samples along the $d_{p}$ parameter.

\section{Isotropic BRDF Measurements}

A key aspect of the PDV parameterization is that isotropic BRDFs are radially symmetric along the perfect reflection vector. Figure 4 illustrates the PDV coordinates along the perfect reflection directions visualized on the hemisphere (3D) and the unit disk (2D). The circles on the unit disk represent level curves on the BRDF lobe, i.e. all samples along a circle will have the same BRDF value. This

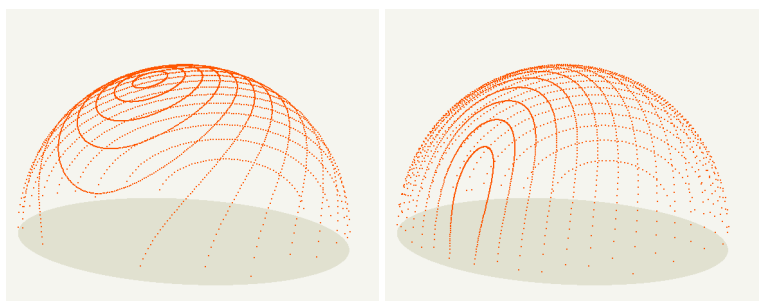

(a) $\theta_{r}=30^{\circ}$

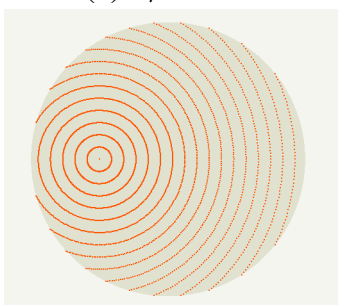

(c) $\theta_{r}=30^{\circ}$ (b) $\theta_{r}=70^{\circ}$

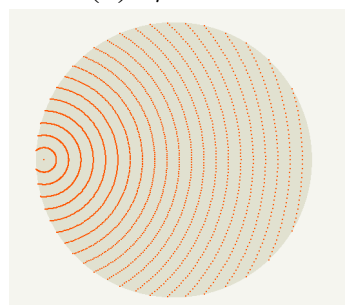

(d) $\theta_{r}=70^{\circ}$
Figure 4. Illustrations of PDV coordinates on hemisphere, (a), (b), and unit disk, (c), (d), with varying $\theta_{r}$. Each coordinate circle is of length $d_{p}=0.044$ apart and rotating $\phi_{p} \in(0,2 \pi)$.

behavior is discussed in the study of the ABC BRDF model by Löw et al. [5]. The PDV parameterization was designed to capture the characteristics of the isotropic BRDFs by exploiting this symmetry. This means that isotropic BRDFs can be described as a 2D function spanned by the two parameters, $\theta_{r}$ and $d_{p}$. Hence we make an assumption that given $\rho_{z 1}\left(\theta_{r}, d_{p}, \phi_{p}=z_{1}\right)$ and $\rho_{z 2}\left(\theta_{r}, d_{p}, \phi_{p}=z_{2}\right)$ then $\rho_{z 1}=\rho_{z 2}$.

Studying the behaviour of measured BRDFs in this 2D representation, we have found that isotropic BRDFs under the logarithmic transform are separable into two $1 \mathrm{D}$ vectors with only very small reconstruction error. We can thus make an assumption that isotropic BRDFs can be decomposed into three univariate functions. Denoting the logarithmically transformed BRDF as $\rho_{t}=\log (\rho+1)$, this can be expressed as follows. For any given point $\left(\theta_{r}, d_{p}, \phi_{p}\right)$

$$
\rho_{t}\left(\theta_{r}, d_{p}, \phi_{p}\right)=F_{1}\left(\theta_{r}\right) F_{2}\left(d_{p}\right) F_{3}\left(\phi_{p}\right),
$$

since $F_{3}\left(\phi_{p}\right)=C$ is constant for any $\phi_{p}$, the BRDF can be described as:

$$
\rho_{t}\left(\theta_{r}, d_{p}, \phi_{p}\right)=F\left(\theta_{r}\right) G\left(d_{p}\right) .
$$

The full BRDF can thus be characterized by measuring the two basis vectors $F\left(\theta_{r}\right)$ and $G\left(d_{p}\right)$ along the $\theta_{r}$ and $d_{p}$ parameter directions respectively as illustrated in Figure 5. $G\left(d_{p}\right)$ can be taken directly from measurements as it describes $F_{2}\left(d_{p}\right) C$ for a fixed parameter value for $\theta_{r}=\theta_{r}^{d p} . F\left(\theta_{r}\right)$ is computed as $F\left(\theta_{r}\right)=F_{m}\left(\theta_{r}\right) / G(x)$, where $F_{m}\left(\theta_{r}\right)$ is the measurement vector along the $\theta_{r}$ direction and $G(x)$ is a ratio factor measured at the intersection of $F_{m}\left(\theta_{r}\right)$ and $G\left(d_{p}\right)$ used to normalize $F\left(\theta_{r}\right)$. This is described in detail below. It is important to note that $F\left(\theta_{r}\right)$ 


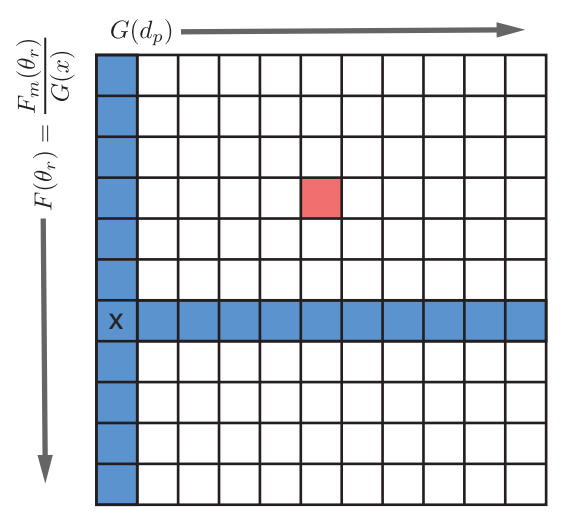

Figure 5. The BRDF matrix illustrates how the two separable basis functions $F\left(\theta_{r}\right)$ and $G\left(d_{p}\right)$ spans the 2D matrix representing the BRDF, and shows that if the blue elements representing $F\left(\theta_{r}\right)$ and $G\left(d_{p}\right)$ are measured, the missing BRDF value in the red element can be computed by using Equation 3 .

and $G\left(d_{p}\right)$ can be measured as a planar slice of the $4 \mathrm{D}$ BRDF.

Measuring $G\left(d_{p}\right)$ (horizontal blocks) is done by fixing a camera direction and moving the light directions over the full $180^{\circ}$ along the plane of incidence illustrated in Figure 6(a). Measuring $F\left(\theta_{r}\right)$ (vertical blocks) is equivalent to move both the light source and the sensor to capture the BRDF data in the perfect reflection directions over the $0-90^{\circ}$ arc as illustrated in Figure 6(b). In order to compute the basis function $F\left(\theta_{r}\right)$ it is necessary to compute the ratio of the actual measurements. This is done by dividing the measured BDRF values, $F_{m}\left(\theta_{r}\right)$, with the measured BRDF value at $G(x)$. In Figure 6(b) this should be thought of as the configuration of the light source and the camera that is the same in the measurement of both $G\left(d_{p}\right)$ and $F_{m}\left(\theta_{r}\right)$, i.e. they represent the same BRDF sample. The location, $x$, of the intersection of $G\left(d_{p}\right)$ and $F_{m}\left(\theta_{r}\right)$ depends on $\theta_{r}^{d p}$ and the configuration of the measurement setup, and can in practice be chosen arbitrarily. In the setup illustrated in Figure 6 it corresponds to $x=0$. This means that for a given $\theta_{r}^{d p}$, the $G(x)$ value corresponds to the direct reflection direction. In Figure 5 the BRDF element corresponding to $G(x=0)$ is the element denoted by $x$.

Isotropic BRDF data is fundamentally represented as a 3D matrix. By using Equation 3, we can estimate the full 2D PDV representation. By using the assumption that the BRDF values along $\phi_{p}$ are constant, the rest of the BRDF data can be estimated using only the reconstructed 2D BRDF data slice. The capture of isotropic BRDFs in the separable PDV parameterization can be carried out using a measurement setup where a light source and sensor move in the same plane. A capture device with one degree of freedom for the light and sensor respectively can be constructed using off-the-shelf components.

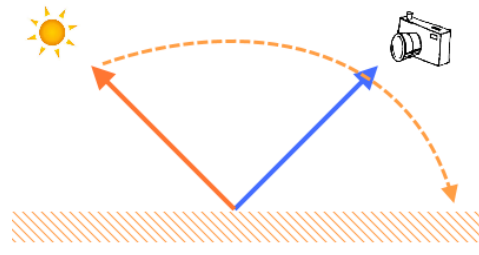

(a) Measuring horizontal BRDF blocks, $G\left(d_{p}\right)$

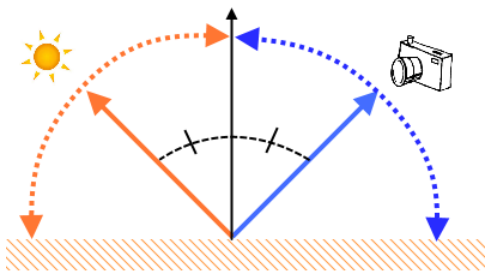

(b) Measuring vertical BRDF blocks, $F\left(\theta_{r}\right)$

Figure 6. The figures illustrate the simple measurements of $G\left(d_{p}\right)$ and $F\left(\theta_{r}\right)$, where (a) measures the shape of the BRDF lobe distribution and (b) the variation of the specular peak over the angular domain.

\section{Results and Discussion}

As our results, we evaluate our BRDF measurement approach using the MERL BRDF data base described in [7]. To numerically evaluate the reconstruction error, we converted all materials in the MERL BRDF data into the PDV parameterization. We then virtually sampled all materials according to the method described in Section 4 and computed the reconstruction error as the difference to the original BRDF data. We also present visual comparisons of synthesized computer graphics images which show the difference between reconstructed and reference materials using the PBRT renderer described by Pharr and Humphreys [10]. BRDF reconstruction error: To numerically compare reconstructions to the reference we use the relative RMS (Root Mean Squared) error. We used the relative RMS error because the absolute error of the specular reflectance may dominate the error of the diffuse reflectance in some regions due to the high dynamic range nature of the BRDF values. The error was computed as:

$$
\text { Error }=\frac{\sqrt{\sum_{i=1}^{N}\left(\frac{\rho_{i, e s t}-\rho_{i, r e f}}{\rho_{i, r e f}}\right)^{2}}}{N},
$$

where $N$ is the number of samples, $\rho_{i, e s t}$ is the reconstructed BRDF of the sampling point $i, \rho_{i, r e f}$ is the reference BRDF of the sampling point $i$.

Each BRDF in the MERL data base was converted to the PDV parameterization at a resolution of $90 \times 90 \times 360$ for the $\theta_{r}, d_{p}$, and $\phi_{r}$ parameters, respectively. For each ma- 


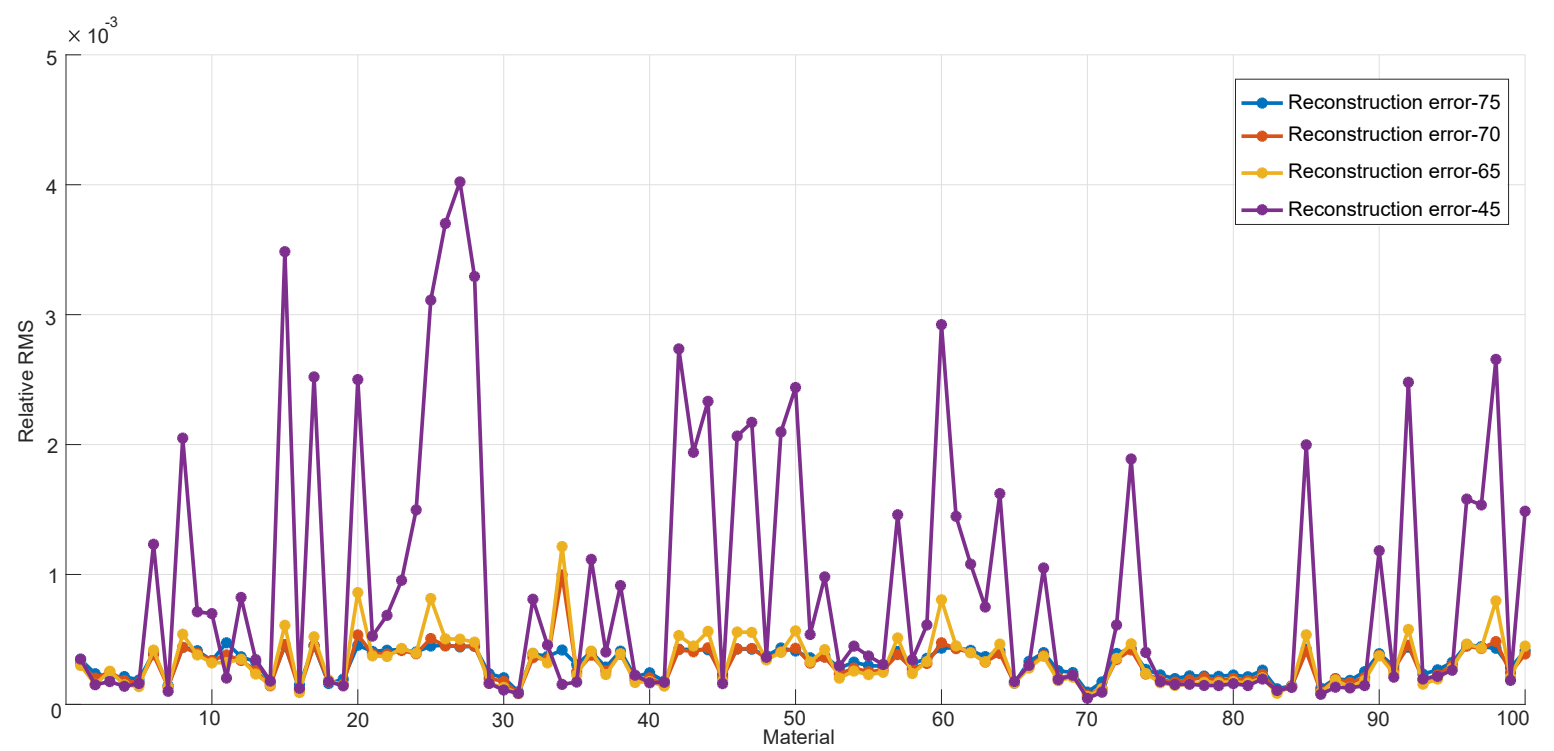

Figure 7. The plots show the reconstruction errors compared to the BRDF references. Each line represents the errors based on specific angles of $\theta_{r}^{d p}=45^{\circ}, 65^{\circ}, 70^{\circ}, 75^{\circ}$.

terial we selected the samples corresponding to the $F\left(\theta_{r}\right)$ and $G\left(d_{p}\right)$ basis functions to reconstruct the full BRDFs by using Equation 3. Thus, we simulated the measurement configuration illustrated in Figure 6. The $G\left(d_{p}\right)$ factor was measured at four different $\theta_{r}$ angles, $\theta_{r}^{d p}=\left\{45^{\circ}, 65^{\circ}, 70^{\circ}\right.$, $\left.75^{\circ}\right\}$. To measure the reconstruction error we, for each BRDF, uniformly sampled $N=3.6$ million samples over the hemisphere in standard spherical coordinates and compared the reconstruction to the reference data in the data base. The plots in Figure 7 show the reconstruction error for all materials in the MERL data base for four $\theta_{r}^{d p}$ angles used in the measurement of $G\left(d_{p}\right)$. The results show that our approach can be used to measure and reconstruct isotropic BRDFs as two separable 1D functions in the PDV parameterization with very small errors. Most reconstruction errors lie below $0.05 \%$, except for when $\theta_{r}^{d p}=45^{\circ}$. The variation between the errors obtained using the different $\theta_{r}^{d p}$ angles could be explained by several reasons including a loss of information in the factorization of the PDV 2D matrix into two 1D functions, noisy data or possibly interpolation artifacts in the conversion from measurements to the Half-Diff representation used in the MERL data base.

Rendering results: Figure 8 shows six examples of reconstructed BRDFs and its luminance difference compared to its reference BRDF. In Figure 8, we selected to use $\theta_{r}^{d p}=70^{\circ}$, as it gives the lowest reconstruction error. The dark blue color of the luminance differences represents the lowest error and the level of white color represents higher error. We see that our method works best on metallic materials or high glossy materials such as gold-metallic-paint3 and black-obsidian. However, our method still performs quite well on diffuse materials such as white-fabric even though the luminance of the reconstructed BRDFs is lower than the luminance of the reference BRDFs.

\section{Conclusions and Future works}

This paper presented a novel approach of isotropic BRDF reconstruction from simple measurements. It was assumed that the PDV parameterization can be used to decompose logarithmically transformed isotropic BRDFs into three univariate functions, and that the PDV parameterization allows us to represent isotropic BRDFs with two parameters. We have shown that a simple measurement setup can recover the dense BRDF data by using our BRDF reconstruction approach. Our simple measurement setup can be used to build efficient measurement devices for isotropic BRDFs. The error plots show that the relative RMS errors between the reconstructed BRDFs and the reference BRDFs are relatively low. Moreover our rendered results of the reconstructed BRDFs are visually very similar to the reference BRDFs.

Future work will be directed towards improving the method to perform better on diffuse materials. We would also like to extend this concept to higher dimensional reflectance data such as SVBRDFs and employ the PDV parameterization in applications where BRDFs need to be characterized in the wild.

\section{Acknowledgements}

This work was partially supported by the Scientific and Technical Research Council of Turkey (Project No: 115E203), the Scientific Research Projects Directorate of Ege University (Project No: 2015/BİL/043). 


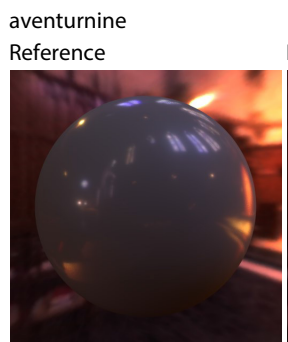

dark-blue-paint

Reference

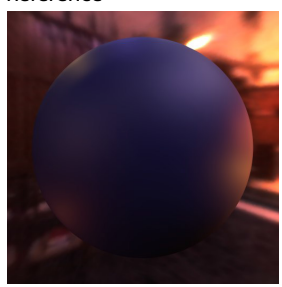

gold-metallic-paint3

Reference

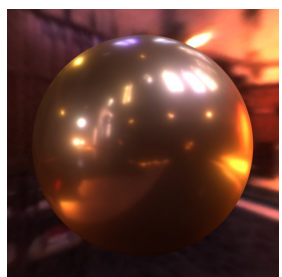

Reconstruction
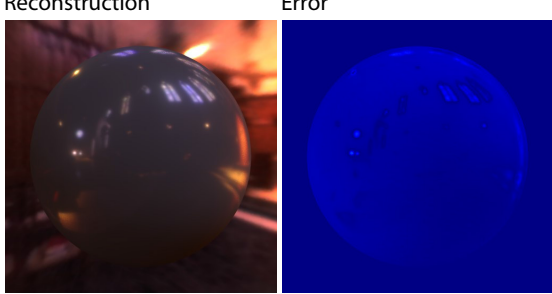

Reconstruction

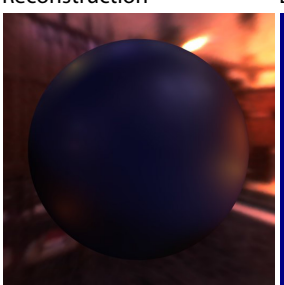

Error

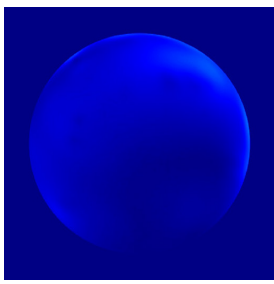

Reconstruction

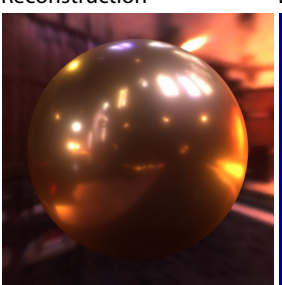

Error

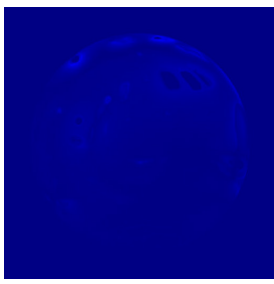

black-obsidian

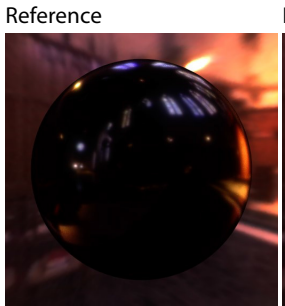

red-metallic-paint

Reference

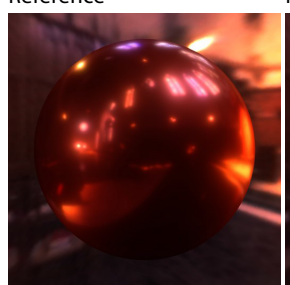

white-fabric

Reference

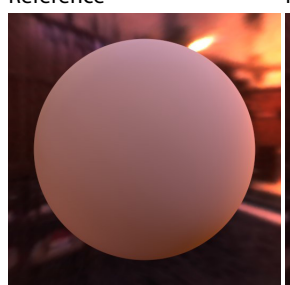

Reconstruction
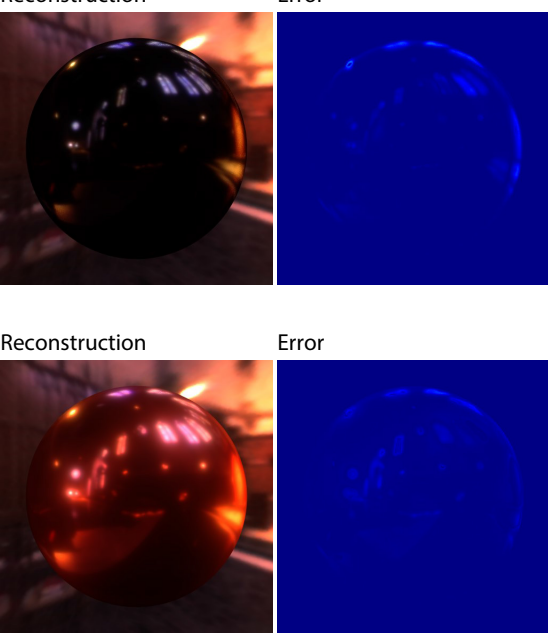

Reconstruction

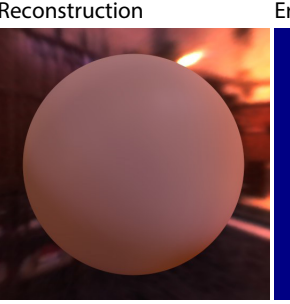

Error

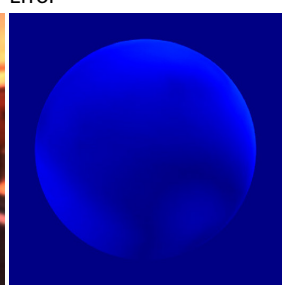

Figure 8. Six materials were rendered to compare between the reference BRDF data and its reconstruction. The third and sixth column show the luminance difference

\section{References}

[1] Light measurement laboratory at cornell university. $\quad$ http://www.graphics.cornell.edu/ research/measure/. Accessed: 2017-08-06. 1

[2] G. Eilertsen, P. Larsson, and J. Unger. A versatile material reflectance measurement system for use in production. In Proceedings of SIGRAD, pages 69-76. Linköping University Electronic Press, 2011. 1

[3] D. Guarnera, G. Guarnera, A. Ghosh, C. Denk, and M. Glencross. BRDF representation and acquisition. Computer Graphics Forum, 35(2):625-650, May 2016. 1

[4] I. Jolliffe. Principal Component Analysis. Springer Verlag, 1986. 2

[5] J. Löw, J. Kronander, A. Ynnerman, and J. Unger. BRDF models for accurate and efficient rendering of glossy surfaces. ACM Transactions on Graphics, 31(1):9:1-9:14, Feb. 2012. 2, 3

[6] S. Marschner, S. Westin, E. Lafortune, and K. Torrance. Image-based measurement of the Bidirectional Reflectance Distribution Function. Applied Optics, 39(16):2592-2600, June 2000. 1

[7] W. Matusik, H. Pfister, M. Brand, and L. McMillan. A datadriven reflectance model. ACM Transactions on Graphics, 22(3):759-769, July 2003. 1, 2, 4

[8] F. E. Nicodemus, J. C. Richmond, J. J. Hsia, I. W. Ginsberg, and T. Limperis. Geometrical considerations and nomencla- ture for reflectance. Monograph, National Bureau of Standards (US), Oct. 1977. 1

[9] J. B. Nielsen, H. W. Jensen, and R. Ramamoorthi. On optimal, minimal BRDF sampling for reflectance acquisition. ACM Transactions on Graphics, 34(6):186:1-186:11, Oct. 2015. 2

[10] M. Pharr and G. Humphreys. Physically Based Rendering, Second Edition: From Theory To Implementation. Morgan Kaufmann Publishers Inc., San Francisco, CA, USA, 2nd edition, 2010. 4

[11] F. Romeiro, Y. Vasilyev, and T. Zickler. Passive reflectometry. In Proceedings of the 10th European Conference on Computer Vision: Part IV, ECCV '08, pages 859-872, Berlin, Heidelberg, 2008. Springer-Verlag. 2

[12] S. M. Rusinkiewicz. A new change of variables for efficient BRDF representation. In G. Drettakis and N. L. Max, editors, Proc. of Eurographics Workshop on Rendering, pages 11-22, Vienna, Austria, 1998. Springer. 1, 2

[13] G. J. Ward. Measuring and modeling anisotropic reflection. Computer Graphics, 26(2):265-272, 1992. (Proc. SIGGRAPH '92). 1

[14] Z. Xu, J. B. Nielsen, J. Yu, H. W. Jensen, and R. Ramamoorthi. Minimal BRDF sampling for two-shot nearfield reflectance acquisition. ACM Transactions on Graphics, 35(6):188:1-188:12, Nov. 2016. 2 